\title{
ORIGINAL ARTICLE \\ Design and analysis of a new medial reciprocal linkage using a lower limb paralysis simulator
}

\author{
M Ahmadi Bani ${ }^{1}$, M Arazpour ${ }^{1}$, F Farahmand ${ }^{2,3}$, S Sefati ${ }^{2}$, M Baniasad ${ }^{2,3}$, SW Hutchins ${ }^{4}$, R Vahab Kashani ${ }^{1}$ \\ and ME Mousavi ${ }^{1}$
}

Study design: A feasibility study on the effect of a new reciprocating orthosis on specific gait parameters for use by people with spinal cord injury.

Objectives: The aim of this study was to design and develop a new medial linkage orthosis (MLO) mechanism incorporating a reciprocating motion and to determine its efficacy in improving specific spatiotemporal, kinematic and kinetic parameters while ambulating when worn by healthy subjects. This was achieved via the use of a lower limb paralysis simulator (LLPS).

Methods: A reciprocating joint with a remote center of motion was designed for use as an MLO. A prototype was fabricated and incorporated into an orthosis and equipped with a saddle to make the reciprocating motion possible. The efficacy of the orthosis was evaluated on four able-bodied healthy subjects who were trained to walk with the MLO attached to the LLPS.

Results: Mean walking speed, stride length, stride time and cadence was $0.09 \pm 0.007 \mathrm{~m} \mathrm{~s}^{-1}, 0.42 \pm 0.01 \mathrm{~m}, 4.89 \pm 0.45 \mathrm{~s}$ and

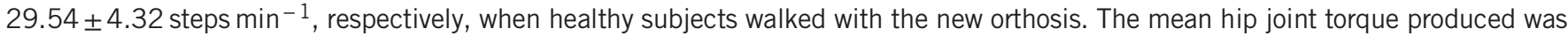
$0.36 \pm 0.13 \mathrm{Nm}$.

Conclusion: In this study a new MLO was designed and fabricated that provided a reciprocating mechanism using a four-bar mechanism to set the virtual axis of the mechanism in a more proximal position than hinge-type joints. Further investigation is currently underway to assess its effect on gait parameters and energy expenditure in paraplegic patients.

Spinal Cord (2015) 53, 380-386; doi:10.1038/sc.2014.193; published online 11 November 2014

\section{INTRODUCTION}

Different approaches have been historically taken with regard to orthotic intervention to help provide standing and walking capability for spinal cord injury (SCI) patients. Mechanical orthoses, ${ }^{1,2}$ powered orthoses $^{3}$ and those using functional electrical simulation ${ }^{4}$ have been used for gait rehabilitation by this patient group. Although powered orthoses and those with functional electrical simulation applied improve gait parameters, they are bulky, heavy and are therefore currently limited for use in a laboratory environment. Hence the most commonly used and functional orthoses to provide standing and walking capability in subjects with SCI are still purely mechanical in design.., 6

There are many different types of mechanical orthoses available, which can be divided into three main categories: ${ }^{7}$ traditional orthoses (hip-knee-ankle-foot orthoses (HKAFOs) and the hip guidance osrthosis (HGO)), reciprocating gait orthoses (the Louisiana State University RGO (LSURGO), the advanced reciprocal gait orthosis (ARGO) and the isocentric reciprocating gait orthosis (IRGO)) and medial linkage orthoses (MLOs) - the Walkabout orthosis (WO), Mooring orthosis and Primewalk orthosis. Excessive energy expenditure and increased loading on the upper limb joints during ambulation are the main reasons for their high rejection rate by paraplegic patients. 8

When compared with different mechanical orthoses, RGOs demonstrate more positive effects in reducing energy expenditure and improving gait parameters than MLOs and HKAFOs because they generate a reciprocal motion at the hip and provide more support for the pelvis and trunk. However, because of their bulky structure and long donning and doffing time, RGOs feel cumbersome for many SCI patients; meaning between $46-54 \%$ of adults with SCI and $61-90 \%$ children with myelomeningocele do not regularly use this type of orthosis. $^{6}$

Owing to the light weight and uncomplicated structure of MLOs, this type of orthosis has been expressed to be more user-friendly than RGOs for walking by SCI patients. ${ }^{910}$ They have removable joints, without a rigid spinal component, and therefore their donning and doffing is potentially easier than RGOs. ${ }^{5,11,12}$ The WO has been shown to reduce energy consumption and improve gait velocity, step length and causes less compensatory motions by paraplegic subjects compared with knee-ankle-foot orthoses (KAFOs) during ambulation ${ }^{13-15}$ but not when compared with the IRGO. ${ }^{16}$ In a comparison between the Primewalk orthosis and the WO on temporal spatial parameters, Saitoh et al. ${ }^{2}$ reported that the Primewalk was more effective than the

${ }^{1}$ Department of Orthotics and Prosthetics, University of Social Welfare and Rehabilitation Science, Tehran, Iran; ${ }^{2}$ Mechanical Engineering Department, Sharif University of Technology, Tehran, Iran; ${ }^{3}$ Research Center of Biomedical Technology and Robotics, Tehran University of Medical Sciences, Tehran, Iran and ${ }^{4}$ University of Salford, IHSCR, Faculty of Health and Social Care, University of Salford, Salford, UK

Correspondence: Dr M Arazpour, Department of Orthotics and Prosthetics, University of Social Welfare and Rehabilitation Science, Kodakyar Street, Daneshjo Boulevard, Evin, Tehran 1985713834, Iran.

E-mail: M.arazpour@yahoo.com

Received 20 March 2014; revised 24 August 2014; accepted 29 September 2014; published online 11 November 2014 
WO. Mean walking speed, cadence and step lengths when using the Primewalk has been shown to be low compared with the IRGO. ${ }^{6,7}$

MLOs are those which are designed to form a simple hinged link between the thigh sections of two KAFOs in an attempt to increase extrinsic stability. However, historically they have not incorporated any components that contribute to walking ability, such as those used in RGOs. The WO has a simple single medial hip joint but does not provide congruency between the mechanical and anatomical hip joints. In an attempt to provide a more advanced MLO, the Primewalk orthosis was introduced that incorporated a sliding medial hip joint ${ }^{2}$ to provide this congruency. However, MLOs do not provide a reciprocating effect when used during walking, which would be advantageous for SCI patients. Fabrication of a more advanced mechanical orthoses, incorporating a combination of the MLO and RGO features, with a view to further improve the structural

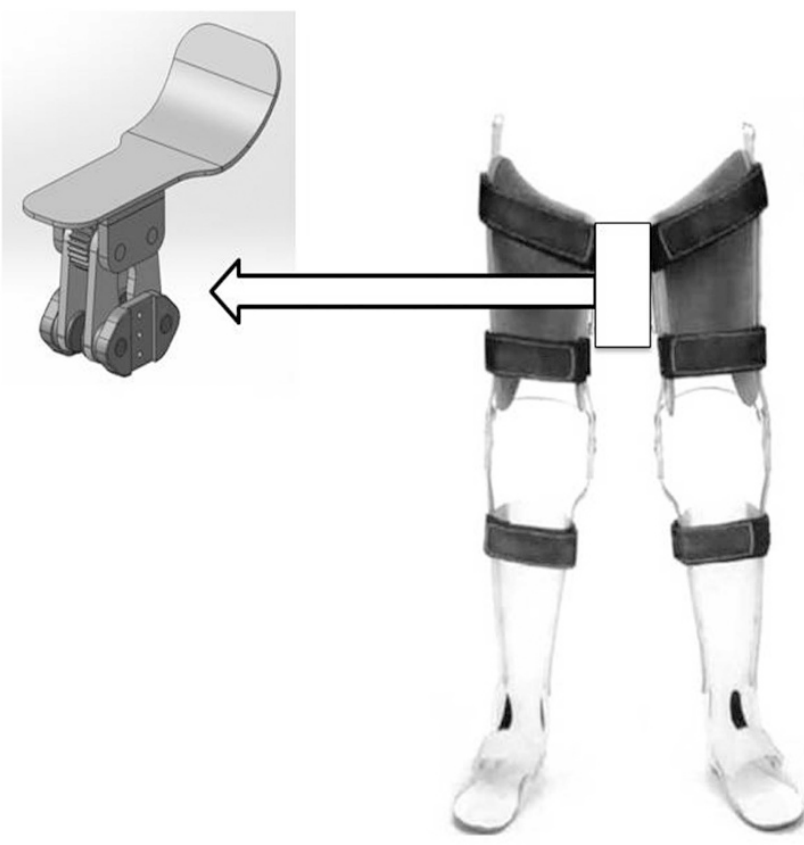

Figure 1 Medial linkage orthosis with reciprocating mechanism.
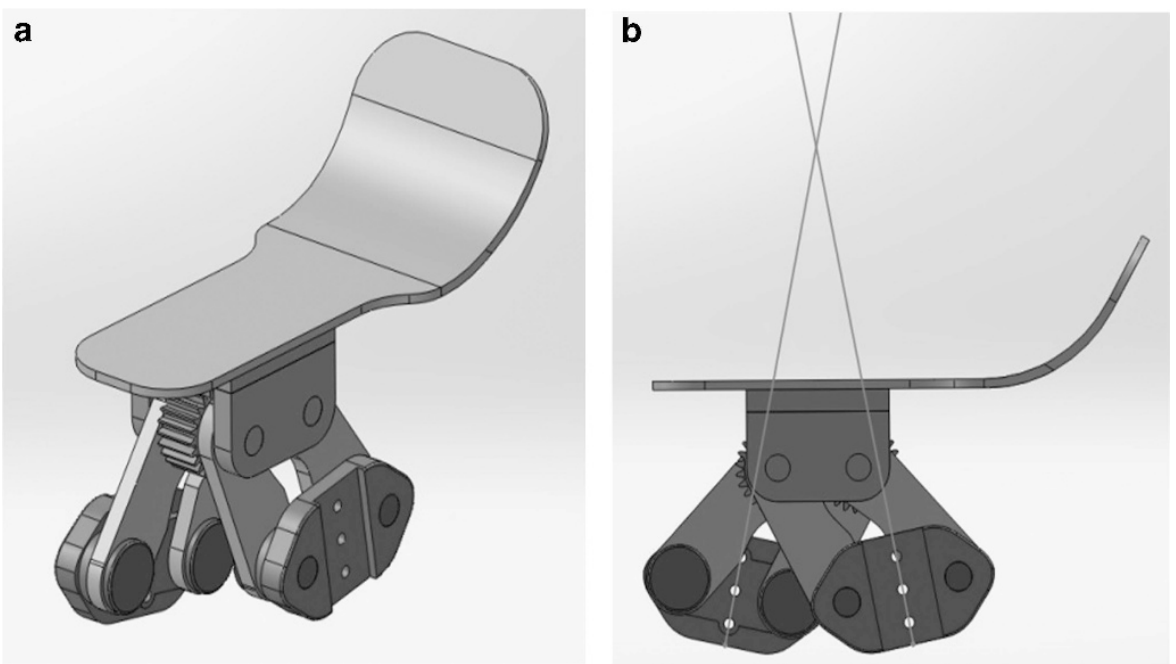

characteristics of the orthosis has been recommended by several studies in this field. ${ }^{17-20}$ The objective of this study was therefore to design and develop a new medial hip joint mechanism incorporating a reciprocating motion and to determine its efficacy on specific spatiotemporal, kinematic and kinetic parameters in healthy subjects initially via the use of a lower limb paralysis simulator (LLPS).

\section{MATERIALS AND METHODS}

\section{Design considerations for a new orthosis}

Kirtly and Mckey ${ }^{21}$ designed and constructed the Walkabout orthosis that was designed with a single medial hip joint. Usability of this orthosis with a wheelchair, its low weight and low price and the convenience offered while donning and doffing made this device a favorable orthosis for walking in subjects with SCI. ${ }^{9,10}$ However, incongruence of the orthotic and anatomical joints has been reported as the main drawback of this device., ${ }^{2,11,12}$ The Mooring and Primewalk orthoses were also designed on the basis of this concept. Although both of these devices possess congruent orthotic and anatomical joints, neither of them has a reciprocating mechanism. ${ }^{2,11}$ The only reciprocal medial linkage HKAFO is the hip and ankle linked orthosis (HALO), but it does not have congruency in orthotic and anatomical joints. ${ }^{22}$

The main concept in developing the new MLO was therefore to incorporate a medial linkage joint with reciprocal motion that could potentially provide cosmesis, acceptance and functional independence alongside improved gait parameters and reduced energy expenditure. ${ }^{9,10}$ A second consideration was matching the anatomical and orthotic joints to further improve gait patterns and potentially decrease the energy cost of walking when donned. ${ }^{2,11}$

The overall design of the new MLO is illustrated in Figure 1. A gearbox joint designed to have a proximal virtual center of motion provides the congruency in orthotic and anatomical joints, as well as the reciprocating movement between the links attached to the gears. The gearbox, on the other hand, is attached to a saddle to receive the motion of pelvis, which enables activation and control of the reciprocating motion.

\section{The medial hip joint mechanism}

The new medial hip joint consists of a perineum gearbox, in which two spur gears are engaged with each other (Figure 2a). One gear is attached to the right side of the joint and the other one to its left side. The two gears move in opposite directions. When the right gear turns backward, it moves the right gear forward and vice versa. Each gear's movement is transmitted to the relevant medial linkage of a KAFO by means of a four-bar mechanism. The dimensions of each link in the four-bar mechanism was chosen carefully to guarantee the congruency of the orthotic and anatomical joints. For instance, in

Figure 2 (a) Gears in prineum gearbox and (b) congruency of anatomical and orthotic joints. 
$30^{\circ}$ of hip flexion, the center of rotation of the orthosis is positioned $60 \mathrm{~mm}$ proximally (Figure $2 \mathrm{~b}$ ) to match the axis of rotation of the patient's hip joint.

During walking, the subject provides stability by putting weight through one leg (the stance leg) and then via posterior tilting of the pelvis the saddle shaped aluminum plate rotates. This rotation is transmitted to the case of the gearbox, turning the gear of the medial linkages backward and that of the swing linkage forward. Thus the rotation of the saddle in extension direction provides hip flexion in the swing leg via the reciprocating mechanism.

\section{Participants}

Four healthy subjects between 18 and 40 years participated in this phase of the study. Exclusion criteria in the selection of participants was based on any known history of orthopedic disease or any other malformation in alignment of the lower limbs, which would affect their ambulation. ${ }^{23}$ Table 1 demonstrates the characteristics of the subjects who volunteered for this study.

\section{Study design and procedure}

To evaluate the efficacy of the new mechanism, the orthosis was tested by the four healthy able-bodied subjects using a LLPS. The aim was to examine the design concepts of the new mechanism when used in a controlled environment. The population of paraplegic patients is small and variant, ${ }^{19,20}$ which makes it difficult to generalize the effect of this new design on the whole paraplegic population. ${ }^{18-20}$ The LLPS simulates the characteristic of paraplegic gait by an able-body person and was introduced for the first time by Johnson et al. ${ }^{19}$ They reported that the LLPS replicated conditions created by a complete spinal lesion at $\mathrm{L}_{1}{ }^{19}$ and it was decided to utilize this technique for an initial evaluation based on the fact that it would only replicate conditions at this spinal level of injury.

The LLPS used in this study was constructed using two vertical aluminum tubes connected to two horizontal axes by fittings. The horizontal axes were connected to the lower ends of the joint. Vertical tubes were connected to the prosthetic feet by pyramid adapters at their distal end. Healthy subjects sat on the saddle of the joints while their lower limbs were dangling. Parallel bars were used to enable the volunteer subjects to balance properly during the walking tests. They were instructed to transfer their weight onto their right leg first and tilt the pelvis posteriorly so that the left leg was propelled forward and vice versa for the other side.

The subjects were trained to walk with the LLPS while using parallel bars during each testing day before being formally evaluated when walking with the orthosis, to enable them to habituate and adapt to wearing it before being tested. Owing to the exhausting nature of ambulating with the LLPS, a 5-min rest period was taken between each test. Five separate sessions were used for each subject (20 in total) that took 8 weeks to complete, because subjects were

Table 1 The characteristics of participated subjects in this study

\begin{tabular}{llccc}
\hline Subject's number & Sex & Age (years) & Height (cm) & Weight (kg) \\
\hline A & F & 28 & 170 & 64 \\
B & M & 29 & 187 & 78 \\
C & M & 34 & 178 & 68 \\
D & F & 27 & 163 & 58
\end{tabular}

Abbreviations: $F$, female; $M$, male. not able to be evaluated in five continual sessions on the same day. Each session typically lasted for $2 \mathrm{~h}$. All tests were performed after the subjects had signed the consent form. The experimental protocol for the study was approved by the Ethical Committee of the University of Social Welfare and Rehabilitation Sciences (USWR).

The subjects were asked to propel the LLPS using their maximum power while they were seated on the saddle. This situation simulates paraplegic walking with crutches. During the test, the subject's right shank was attached to a load cell (Bongshin S beam load cell, Model DBBP $100 \mathrm{~kg}$, Seongnam, Korea) to measure the maximum force they could generate by their leg. After $20 \mathrm{~s}$ of rest, they propelled their right leg using the maximum force they could exert on the load cell's cable during the next $20 \mathrm{~s}$ and then rested again for $20 \mathrm{~s}$. This test was repeated for three trials. Two markers were attached to the thigh and shank and two markers were attached to the cable for detecting the angle between the cable and the leg. The torque produced by the system was computed using the following formula:

$$
T=\frac{F \times L \times \cos \theta}{\mathrm{BW}}
$$

where $T$ is the normalized torque produced by the system, $F$ is the force recorded by load cell, $L$ is the distance between the point where the load cell was attached to the LLPS leg and the center of rotation of the joint and $\theta$ is the angle between the cable and leg of the LLPS. The resulting torque was divided by the subject's body weight (BW) for normalization.

In the next set of experiments, gait analysis was performed. Data collection was performed using a Vicon digital motion capture system (Oxford Metrics, UK, 460), via six cameras (Vicon, Infrared, Oxford, UK) with $100 \mathrm{HZ}$ frequency in three trials at a biomechanics laboratory. The marker placements were based on a conventional marker set on the trunk (left and right acromioclavicular joint, seventh cervical vertebrae and sixth thoracic vertebrae), the pelvis (sacrum and both anterior superior iliac spine (ASIS)), the posterior of saddle plate (the top edge, plus a position $5 \mathrm{~cm}$ lower than its superior edge on left and right edge) and the LLPS (both thighs and shanks, plus the heel, fist and fifth metatarsals). Rest periods were given if the subjects required them between the trials. The mean of three trials was used for plots of trunk motion, hip flexion, saddle movement and spatiotemporal parameters were calculated by Matlab (MathWorks, Natick, MA, USA) software.

\section{Statistical analysis}

The normal distribution of all variables was using the Kolmogorov-Smirnov test. SPSS statistical software version 16.0 (SPSS Inc., Chicago, IL, USA) was used for analysis of the data. The level of significance was set at 0.05 .

\section{RESULTS}

\section{Spatiotemporal parameters:}

Table 2 indicates the spatiotemporal parameters of walking for each subject. The mean of speed of walking was $0.09 \pm 0.007 \mathrm{~m} \mathrm{~s}^{-1}$ during ambulation with LLPS. The mean of stride length and time were $0.42 \pm 0.01 \mathrm{~m}$ and $4.89 \pm 0.45 \mathrm{~s}$, respectively. The mean of cadence was $29.54 \pm 4.32$ steps $\min ^{-1}$, when healthy subjects walked with LLPS in this study.

Table 2 Mean and s.d. of the spatiotemporal, kinematic parameters and the hip joint torque during swing phase of walking in healthy subjects with LLPS

\begin{tabular}{llccc}
\hline Subject's number & Stride length $(\mathrm{m})$ & Stride time $(\mathrm{s})$ & Cadence $\left(\right.$ step min $\left.{ }^{-1}\right)$ & Velocity $\left(\mathrm{ms}^{-1}\right)$ \\
\hline A & $0.39 \pm 0.03$ & $5.49 \pm 0.03$ & $22.37 \pm 4.08$ & $0.06 \pm 0.01$ \\
B & $0.41 \pm 0.01$ & $2.41 \pm 0.05$ & $50.76 \pm 9.09$ & $0.17 \pm 0.006$ \\
C & $0.40 \pm 0.0008$ & $5.99 \pm 0.34$ & $21.78 \pm 1.91$ & $0.21 \pm 0.01$ \\
D & $0.49 \pm 0.02$ & $5.65 \pm 0.46$ & $23.26 \pm 1.38$ & 0.004 \\
Mean and s.d. & $0.42 \pm 0.01$ & $4.89 \pm 0.45$ & $29.54 \pm 4.32$ & $0.09 \pm 0.004$ \\
\hline
\end{tabular}

Abbreviation: LLPS, lower limb paralysis stimulator. 

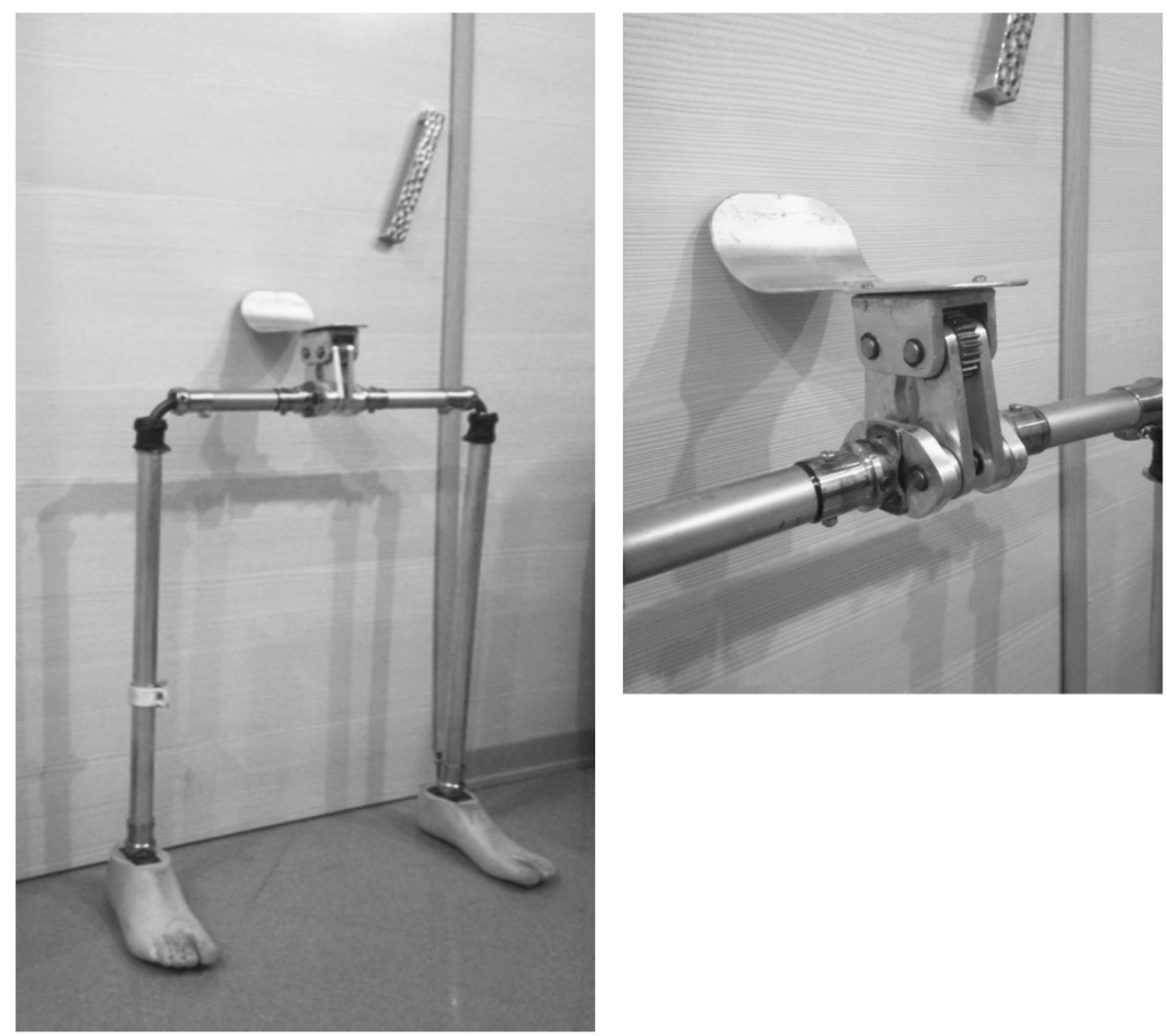

Figure 3 LLPS used in this study and joint mechanism.

\section{Hip joint torque}

Table 2 also shows the hip joint torques produced by the system. The torque that is produced in the swing phase of walking by a normal person is $2 \mathrm{Nm} \mathrm{kg}^{-1}$ when normalized by body weight. The mean of the hip joint torque produced by the MLO orthosis of the present study was $0.36 \pm 0.13^{\circ}$, when healthy subjects walked with LLPS.

\section{Kinematic parameters}

Figure 3 shows the means of the hip joint angle and the trunk angle while walking with LLPS. In comparison with normal gait, both angles (hip and trunk) showed reduction in flexion and extension rates.

Subjects 3 and 4 demonstrated normal patterns of hip joint kinematics during stance and swing phase. These two subjects had $\sim 5^{\circ}$ of flexion at heel strike. This pattern is similar to normal walking and the hips demonstrated $12^{\circ}$ of extension at the end of stance phase. Subjects 1 and 2 had $\sim 2^{\circ}$ of flexion in heel strike. The flexion of the hip joint altered to $11^{\circ}$ of extension at $45 \%$ of the gait cycle. These subjects had shorter stance percentages compared with normal patterns.

Sagittal plane kinematics of the trunk is shown in Figure 4. Sagittal plane motion of the trunk closely followed that of the pelvic motion and demonstrated a sinusoidal pattern with a period equal to the step cycle (that is, half a gait cycle).

Sagittal plane kinematics of the saddle plate is also illustrated in Figure 3. Its pattern was sinusoidal, the first extension of the saddle plate is the result of the first posterior pelvic tilt leading to propulsion of the left leg and the second one is due to the second posterior pelvic tilt, driving the right leg forward. Subjects 3 and 4 had larger ranges of motion in the saddle plate and higher ranges of hip flexion in comparison with subjects 1 and 2 .

\section{DISCUSSION}

In this study, to improve the efficacy of MLOs, a new orthosis that provided a reciprocating effect with congruency between anatomical and mechanical hip joints was designed and fabricated for walking in patients with SCI. To our knowledge, it is the first medial linkage that provides reciprocal motion as well as congruent anatomic and orthotic joints, which is sensitive to pelvic motion. The new joint was attached to an LLPS to simulate walking patterns, previously reported by SCI subjects in a laboratory setting. In a previous study, it has been shown that the LLPS can model characteristic features of RGO-assisted gait by SCI patients, albeit at one specific spinal level of injury. ${ }^{19}$

By tilting the pelvis posteriorly and transferring the body's line of gravity posterior to that of the premium gearbox, LLPS users were able to tilt the saddle downward and make the LLPS' leg to move forward. The hip joint pattern of motion demonstrated that the newly developed joint could move the lower limb forward by posterior pelvic tilt. Therefore it could feasibly be used by patients with lower limb paraplegia, yet a stable pelvic girdle. The results showed a $0.42 \mathrm{~m}$ stride length and $0.09 \mathrm{~m} \mathrm{~s}^{-1}$ walking speed, which is higher than that reported in previous studies. However, the most important advantage of the new joint over current RGOs is its medial placement that could provide less bulk and less additional weight for the patient and decrease brace rigidity and possibly increase patient acceptance.

Walking with the LLPS and the new medial hip joint demonstrated production of $>0.3 \mathrm{Nm} \mathrm{kg}^{-1}$ of hip torque, (Figure 3 ) which is larger than that previously reported for RGO hip torque (which has been 
a

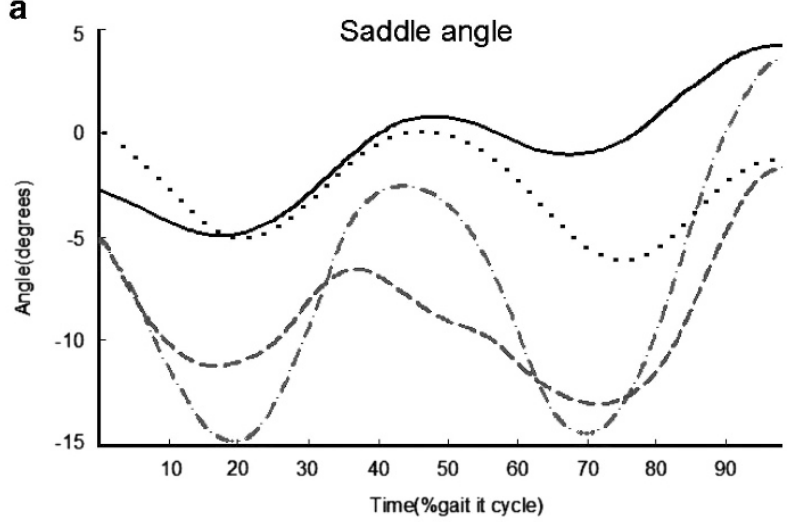

b

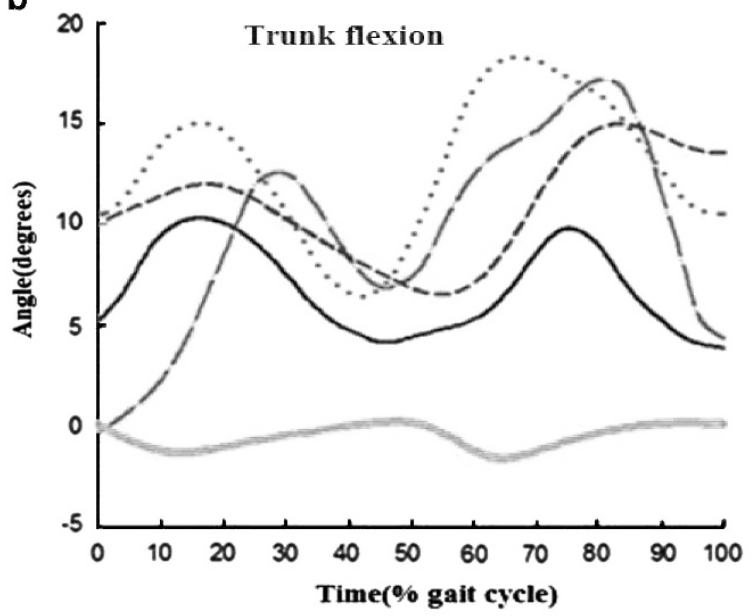

C

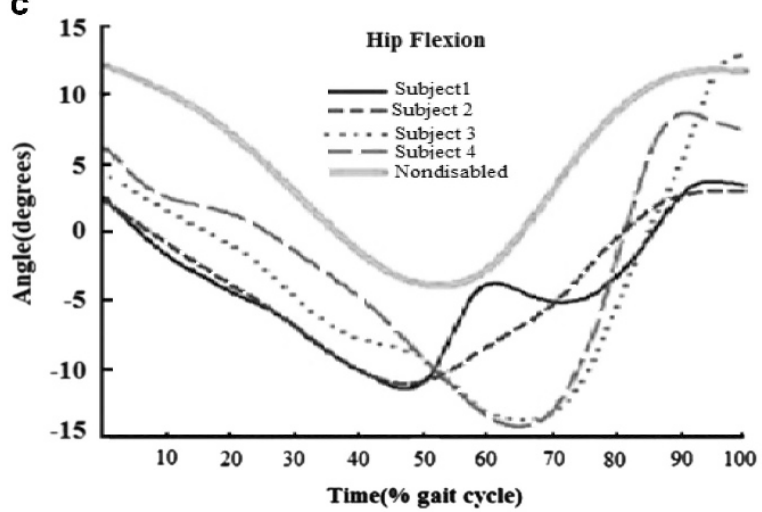

Figure 4 Mean data for LLPS users. (a) Saddle extension (b) Trunk flexion angle. (c) Hip flexion angle.

$<0.3 \mathrm{Nm} \mathrm{kg}^{-1}$ ). Dall et al. ${ }^{18}$ reported that in four subjects out of the six who were evaluated, no movement was found in the front cable that was used to create hip flexion and the torques measured in the other two subjects were very low and only made a small contribution to lower limb flexion. In this study, the subjects produced more torque than the results reported by Dall et al., ${ }^{18}$ but it should be noted that in Dall et al.'s study, SCI patients with higher levels of injury had participated than healthy subjects participating in this study and this may be the reason for the high torque readings demonstrated. Also, Johnson et al. ${ }^{19}$ calculated hip joint torque for extension and reported it to be between 0 and $0.3 \mathrm{Nm} \mathrm{kg}^{-1}$. In the present study, however, we measured the exact flexion torque and found it to be $0.3 \mathrm{Nm} \mathrm{kg}^{-1}$ while standing. In an IRGO, the force transition from one side to another can be wasted during transference, whereas in this new system the gears transition would have reduced this waste of energy. Our results were calculated in standing, therefore hip torque during ambulation was not calculated.

With regard to spatiotemporal parameters, Table 3 demonstrates the mean data obtained when SCI subjects have used mechanical orthoses in previous studies. Walking speed, stride length and cadence have all been affected depending on the orthosis used. The IRGO and HALO are the most effective mechanical orthoses in improving these parameters. A reciprocating mechanism is responsible in providing improvement in spatiotemporal parameters when used by SCI patients, whereas a movable ankle joint and a connection between the hip joint and ankle joint influences gait parameters in the HALO. The mean walking speed, stride length, stride time and cadence was $0.09 \pm 0.007 \mathrm{~m} \mathrm{~s}^{-1}, 0.42 \pm 0.01 \mathrm{~m}, 4.89 \pm 0.45 \mathrm{~s}$ and $29.54 \pm 4.32$ steps $\min ^{-1}$, respectively when healthy subjects walked with the new orthosis in this study.

There are many mechanical orthoses that provide standing and walking in paraplegic patients. MLOs are the new generation of this type of orthoses that have superiority in wheelchair compatibility and ease of donning/doffing compared with other types of mechanical orthoses. ${ }^{9,10}$ Although joint mobility and psychological benefits have been reported as the main positive effects of using Walkabout system in SCI patients; ${ }^{24}$ the Walkabout system has a problem related to its mechanical design, it does not possess a congruent anatomical and orthotic hip joint. Development of Primewalk and Moorong MLO was undertaken to potentially solve the drawbacks associated with the Walkabout's mechanical system, ${ }^{2,11}$ and this has been also attempted in this new MLO. The discrepancy in height between the physiological hip joint and orthotic joint can reduce the effective length of the lower extremity in the MLOs, as well as compensatory rotational pelvic movement in the horizontal plane. ${ }^{12}$ While using the Walkabout system for walking, paraplegic patients had to produce more arm movements to accommodate this effect and to achieve balance. On the basis of this point, less energy consumption has been reported for Moorong MLO in comparison with the Walkabout system during walking of SCI patients on various terrains. ${ }^{11}$ In addition, in comparing the energy expenditure of Walkabout system and IRGO, Harvey et al. ${ }^{9}$ found that the physiological cost index and oxygen cost were significantly greater for Walkabout in patients with paraplegia. ${ }^{16}$ According to these studies, walking with this new orthoses may decrease energy consumption because the position of the orthotic hip joint is set close to that of the physiological hip joint. Less compensatory motion during walking has been reported when using the MLO compared with a KAFO in SCI subjects. ${ }^{13}$ It can therefore be hypothesized that energy consumption may decrease in SCI patients when using the new MLO, and therefore this parameter should be evaluated on SCI patients in future studies.

The clinical implications of this study are that, following further development of the device described in this study, paraplegic patients may be able to ambulate with an orthosis incorporating a reciprocating mechanism in a medial link-type device. It includes a reciprocating mechanism in addition to providing congruency between anatomical and orthotic joints, and therefore seems to be advantageous over the currently available medial linkage orthoses. In fact, the method of implementing the reciprocating mechanism in our orthosis might be even more effective than that of the current RGO designs.

In this study the new joint design was evaluated using an LLPS but a future study should evaluate its efficacy with SCI patients with 
Table 3 The mean of the temporal spatial parameters in using of mechanical orthoses in the $\mathrm{SCl}$ patients

\begin{tabular}{|c|c|c|c|c|c|}
\hline Author & Subject & Intervention & \multicolumn{3}{|c|}{ Gait parameter } \\
\hline \multirow[t]{2}{*}{ Harvey et al. ${ }^{16}$} & & IRGO & - & - & $11.5 \pm 2.3$ \\
\hline & & WA & - & - & $5.2 \pm 1.3$ \\
\hline Middleton et al. ${ }^{11}$ & $1 \mathrm{C} 6$ & Mooring & - & - & 8.0 \\
\hline Genda et al. ${ }^{22}$ & & PW & 1.00 & 58.9 & 0.48 \\
\hline \multirow[t]{2}{*}{ Onogio et al. ${ }^{12}$} & 7 (T4-10) & PW & 0.43 & 48.0 & 0.17 \\
\hline & & WA & 0.36 & 40.8 & 0.12 \\
\hline
\end{tabular}

different levels of injury, because a higher level may produce different effects. In this study, the effects of the new orthosis on gait parameters were not analyzed by paraplegic patients and further studies are planned to examine the efficacy of this new orthosis on walking of SCI patients. Comparison between this MLO and other MLOs (for example, the Walkabout and Primewalk), plus a comparison between the IRGO and this new type of MLO will also be beneficial.

\section{Limitation of study and future design of study}

In this study, the force applied on the saddle that is helpful to determine the amount of force that is needed for driving LLPS forward was not evaluated. Subjects in this study walked with the aid of parallel bars as the LLPS does not provide trunk support. The laboratory did not possess a portable force plate system to measure the force applied in upper and lower limbs. In addition, it was not possible to directly compare the results of this study with other previous studies as this study did not have a directly comparable cohort of subjects.

In future studies, this new orthosis should be evaluated on patients with different levels of paraplegia and also complete and incomplete injury to discover more information about its efficacy in altering gait parameters, as well as its effect on energy consumption, functional independency and the forces applied on crutches and lower limbs when ambulating. It should also be compared with other available reciprocal orthoses, as well as medial linkage orthoses.

\section{CONCLUSION}

In this study a new MLO was designed and fabricated which provided a reciprocating mechanism with congruency between anatomical and mechanical hip joints. This was acheived by using a four-bar mechanism that set the virtual axis of the orthotic hip joint in a more proximal position than simple hinge-type joints. The reciprocating mechanism was supported by using a gearbox-saddle complex that provided a reciprocal motion. Further investigation is currently underway to assess its effect on gait parameters and energy expenditure in paraplegic patients.

\section{DATA ARCHIVING}

There were no data to deposit.

\section{CONFLICT OF INTEREST}

The authors declare no conflict of interest.

\section{ACKNOWLEDGEMENTS}

We thank Dr Steven A Gard for his advice and comments. We also thank the Iran National Science Foundation for financial support (grant number 92040942) for this research.

1 Motlock W. Principles of orthotic management for child and adult paraplegia and clinical experience with the isocentric RGO. In: Proceeding of 7th World Congress of the International Society in Prosthetic and Orthotics; 28 June-3 July 1992; Chicago, IL, USA, p. 28

2 Saitoh E, Baba M, Sonoda S, Tomiya Y, Suzuki M, Hayashi A. A new medial single hip joint for paraplegic walkers. In: Ueda S, Nakamura R, Ishigami S. (eds) The Eighth World Congress of International Rehabilitation Medicine Association. Monduzzi Editore: Bologna, Italy, 1997, pp 1299-1305.

3 Arazpour M, Chitsazan A, Hutchins SW, Ghomshe FT, Mousavi ME, Takamjani EE et al. Design and simulation of a new powered gait orthosis for paraplegic patients. Prosthet Orthot Int 2012; 36: 125-130.

4 Jailani R, Tokhi M, Gharooni S. Hybrid orthosis: the technology for spinal cord injury. J Appl Sci 2010; 10: 2785-2792.

5 Karimi MT. Functional walking ability of paraplegic patients: comparison of functional electrical stimulation versus mechanical orthoses. Eur J Orthop Surg Traumatol 2012; 23: 631-638.

6 Arazpour M, Bani MA, Hutchins SW. Reciprocal gait orthoses and powered gait orthoses for walking by spinal cord injury patients. Prosthet Orthot Int 2013; 37: $14-21$.

7 Ahmadi Bani M, Arazpour M, Farahmand F, Mousavi ME, Hutchins SW. The efficiency of mechanical orthoses in affecting parameters associated with daily living in spinal cord injury patients: a literature review. Disabil Rehabil Assist Technol. e-pub ahead of print 2 January 2014.

8 Tashman S, Zajac F, Perkash I. Modeling and simulation of paraplegic ambulation in a reciprocating gait orthosis. J Biomech Eng 1995; 117: 300-308.

9 Harvey LA, Newton-John T, Davis GM, Smith MB, Engel S. A comparison of the attitude of paraplegic individuals to the walkabout orthosis and the isocentric reciprocal gait orthosis. Spinal Cord 1997; 35: 580-584.

10 Harvey LA, Smith MB, Davis GM, Engel S. Functional outcomes attained by T9-12 paraplegic patients with the walkabout and the isocentric reciprocal gait orthoses. Arch Phys Med Rehabil 1997; 78: 706-711.

11 Middleton J, Fisher W, Davis G, Smith R. A medial linkage orthosis to assist ambulation after spinal cord injury. Prosthet Orthot Int 1998; 22: 258-264.

12 Onogi K, Kondo I, Saitoh E, Kato M, Oyobe T. Comparison of the effects of sliding-type and hinge-type joints of knee-ankle-foot orthoses on temporal gait parameter in patients with paraplegia. Jpn J Compr Rehabil Sci 2010; 1: 1-6.

13 Arazpour M, Bani MA, Hutchins SW, Sayyadfar M. The Araz medial linkage orthosis: a new orthosis for walking in patients with spinal cord injury: A single patient study. Prosthet Orthot Int 2014; 38: 155-159.

14 Cliquet Jr A, Baxendale R, Andrews B. Paraplegic locomotion and its metabolic energy expenditure. Compr Neurol Rehabil 1989; 3: 139-146.

15 Saitoh E, Suzuki T, Sonoda S, Fujitani J, Tomita Y, Chino N. Clinical Experience With A New Hip-Knee-Ankle-Foot Orthotic System Using A Medial Single Hip Joint for Paraplegic Standing and Walking1. American J Phys Med Rehabil 1996; 75: 198.

16 Harvey L, Davis G, Smith M, Engel S. Energy expenditure during gait using the walkabout and isocentric reciprocal gait orthoses in persons with paraplegia. Arch Phys Med Rehabil 1998; 79: 945-949.

17 Bernardi M, Canale I, Castellano V, Di Filippo L, Felici F, Marchetti M. The efficiency of walking of paraplegic patients using a reciprocating gait orthosis. Paraplegia 1995; 33: 409-415.

18 Dall P, Müller B, Stallard I, Edwards J, Granat M. The functional use of the reciprocal hip mechanism during gait for paraplegic patients walking in the Louisiana State University reciprocating gait orthosis. Prosthet Orthot Int 1999; 23: 152. 
19 Johnson W, Fatone S, Gard S. Modeling the walking patterns of reciprocating gait orthosis users with a novel lower limb paralysis simulator. In: Conf Proc IEEE Eng Med Biol Soc; 30 August-3 September 2011. Boston, MA, USA, 2011, pp 7841-7844.

20 Johnson WB, Fatone S, Gard SA. Walking mechanics of persons who use reciprocating gait orthoses. J Rehabil Res Develop 2009; 46: 435-446.

21 Kirtley C, McKay S. Total design of the walkabout, a new paraplegic walking orthosis. Proceedings, seventh world congress of ISPO 1992, Chicago: Illinois, USA.
22 Genda E, Oota K, Suzuki Y, Koyama K, Kasahara T. A new walking orthosis for paraplegics: hip and ankle linkage system. Prosthet Orthot Int 2004; 28: 69-74.

23 Arazpour M, Ahmadi F, Bani MA. Hutchins SW, Bahramizadeh M, Ghomshe FT et al. Gait evaluation of new powered knee ankle foot orthosis in able-bodied persons: a pilot study. Prosthet Orthot Int 2014; 38: 39-45.

24 Middleton J, Yeo J, Blanch L, Vare V, Peterson K, Brigden K. Clinical evaluation of a new orthosis, the'walkabout', for restoration of functional standing and short distance mobility in spinal paralysed individuals. Spinal Cord 1997; 35: 574-579. 